Research Article

\title{
Digitally Synthesized Alternative Current Sinusoidal Waveform for Resistive Sensor Measurements
}

\author{
K. N. Tarchanidis* and A. T. Soilemes \\ Department of Petroleum Technology, University of Kavala Institute of Technology, Ag. Loukas, 654 04, Kavala, Greece.
}

Received 2 August 2010; Accepted 20 January 2011

\begin{abstract}
This paper presents a circuit that has the ability to provide a sinusoidal current waveform. The produced waveform is alternative, so it takes positive and negative values. This circuit comprises a microcontroller, a DAC with current output and a fast electronic switch. The sine wave samples are stored in the microcontroller's data memory and are applied to the DAC's digital 8-bit input. The complementary output currents are connected alternatively through the electronic switch to the ground. At pre-calculated timing the microcontroller changes the polarity of the switch, allowing the DAC's output, as alternative current, to flow through the resistive sensor (load), ending through the switch to the ground. Another microcontroller acting as data acquisition chip, at the positive peaks is sampling the voltage across the load. These measurements are transferred through the serial port to a PC, where the resistance is calculated and presented on screen.
\end{abstract}

Keywords: Digital Sine wave, Current Source, Resistive Sensors, Microcontroller, Embedded Application.

\section{Introduction}

The digitally synthesized sine waveform (Direct Digital Synthesis, DDS) is a well-known method and has been applied to many embedded applications [1]. This technique can be used to create a positive digital sine waveform. This DC offset produces numerous problems when the parasitic capacitance is not negligible. The DC offset charges the capacitance and the data acquisition circuitry is misled to false measurements. This problem is more pronounced at very small voltage measurements, since the parasitic capacitances are charged and the measurement is actually not accurate, due to the difficulty to discriminate the ac from the dc component.

The obvious solution to the problem is the creation of an alternative sine waveform. The parasitic capacitance will charge, discharge and charge at the opposite polarity. The appropriate timing for voltage measurement is at the discharged state. The digitally synthesized sine has the advantage to produce signals (quantized samples) at any phase of a sine circle. Normally for that kind of waveform a symmetrical power supply would be required. In this paper, the pro-posed digital sine uses a single power supply and achieves to create an alternative current waveform. This was made possible by redirecting the current flow through the load giving the sense of a floating current source.

The applications of this alternative digital sine are limitless,

*E-mail address: ktarch@teikav.edu.gr

ISSN: 1791-2377 (C) 2011 Kavala Institute of Technology. All rights reserved. starting of all the resistive sensors, [2], [3] to any possible resistance measurement [4].

A microcontroller based data acquisition system with 8 bit resolution, is used to sample the voltage drop across the load and to transmit the collected data to the PC through the RS232 serial port.

\section{Digital Synthesis}

\subsection{Generation of sine waveforms}

A sine waveform can be approximated by $\mathrm{N}$ equally-spaced samples per cycle, as shown in Fig. 1. It is well-known that according to the Nyquist sampling theorem that $\mathrm{N}$ must be equal or greater than two. In our case, $\mathrm{N}$ is much larger than two in order to reduce the spectral content of the produced sine wave. That means that the sine wave is pure enough without the need of extra filtering [5]. Each sample has a time duration $\tau$, equal to the period of a higher frequency clock pulses, and amplitude which may be expressed as:

$$
\sin \left(\frac{2 \mathrm{k} \pi}{\mathrm{N}}\right) \text {, }
$$

where $\mathrm{k}=0,1,2,3 \ldots$ 


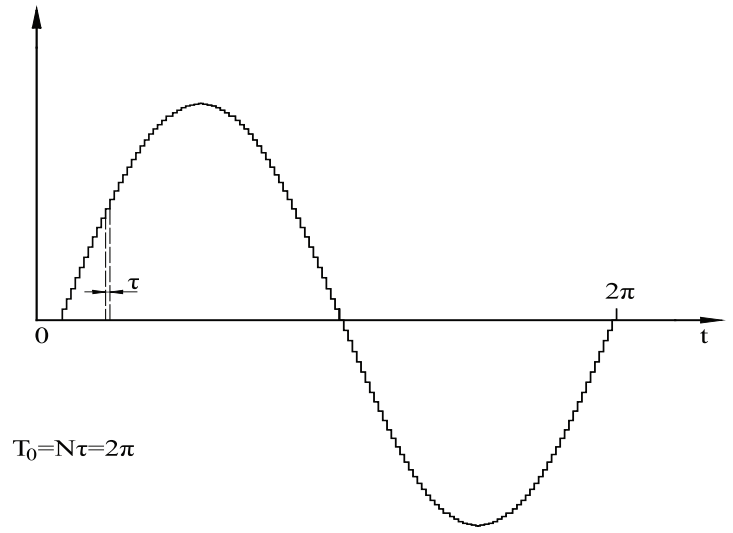

Figure 1. A sine waveform approximated by $\mathrm{N}$ equally-space samples per cycle

As a consequence, the sine waveform period is $\mathrm{T}_{0}=\mathrm{N} \tau$, i.e. the fundamental frequency of the sine wave is $\mathrm{N}$ times smaller than the clock frequency. The digitally - generated sine wave function is given by:

$$
f(t)=\sin \left(\frac{2 \pi}{N \tau}\right) t=\sin \left(\frac{2 k \pi}{N}\right),
$$

where $\mathrm{t}$ is given by: $\mathrm{t}=\mathrm{k} \tau$ and $\mathrm{k}=0,1,2,3 \ldots[6]$

\subsection{Digital Values generation}

In Fig. 2 the circuit diagram for the digital sinusoidal current source is presented. Photographs 1 and 2 show the circuit implementation.

The sine function values were calculated and the round up integer was deducted by the resulting real numbers. These values

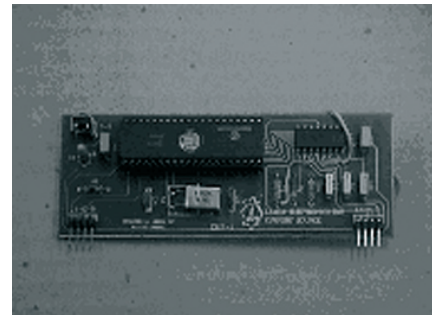

Photo 1. Alternative Sinusoidal Current Source (top)

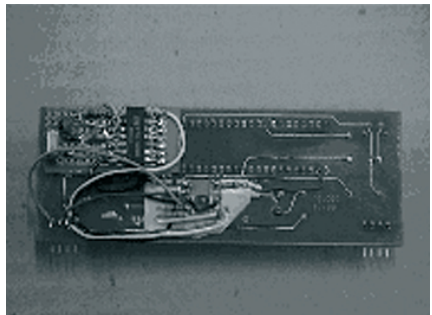

Photo 2. Alternative Sinusoidal Current Source (bottom) were stored in the microcontroller's EPROM program and data memory. A microcontroller program was written to output those values sequentially on one of its output ports (Port B). That port is directly connected to the 8-bit DAC input.

Since the sine discrete values are sequentially provided to the $\mathrm{DAC}$, the final sine frequency will depend on the time intervals between each new value on the port. Consequently, the final frequency is constrained by the program steps involved to complete a full sine cycle. Furthermore, the execution time of those steps depends on the microcontroller's clock. In this project a $4 \mathrm{MHz}$ microcontroller clock was used. The resulting sine waveform frequency was approximately $30 \mathrm{kHz}$.

Furthermore, the output amplitude is a function of the internal voltage reference of the DAC. The sine amplitude proved to be stable since the values were prestored in the microcontroller's EPROM data memory. The stored sine values were in total 132 and were divided in two groups, according to the quadrant. The values for the first two quadrants are the results of the sine function $\mathrm{f}(\mathrm{x})=\sin \mathrm{x}$, where $0<\mathrm{x}<\pi$. In our case $\mathrm{x}$ can have 66 discrete values from 0 to $\pi$ rads.

When the output reaches the positive maximum, an indication bit flag is set, commutating (toggling) from zero to one (0-1),

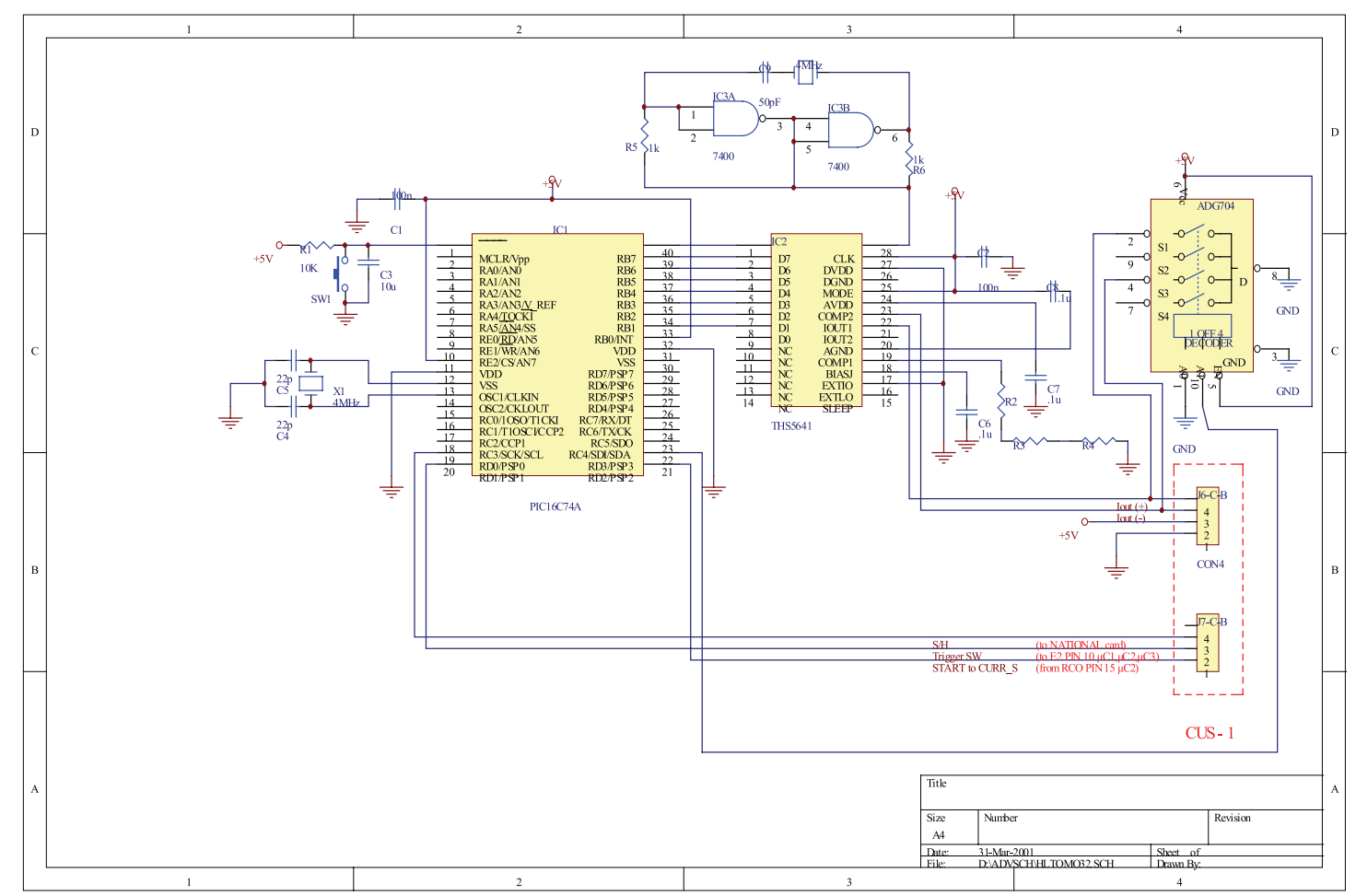

Figure 2. Circuit Diagram 
on bit 0 of the microcontroller's Port C. After three program steps, the bit flag resets to zero (0). This flag is used by the data acquisition circuitry to sample the voltage drop across the load. The output peak current value is stable and is used for the A/D sampling, since it will provide positive potential difference.

Since only positive samples can be generated, the actual waveform stored in the microcontroller's memory is like the one shown in Fig. 3. It is obvious that a DC correction is needed every other half period, i.e. for the last two quadrants a dc offset is added to the calculated samples value. This offset will result an output of the 8-bit maximum (FFh) for the lowest and highest $\mathrm{x}$ values $(\pi$, $2 \pi$ ) and a minimum (00h) for $\mathrm{x}=3 \pi / 2$.

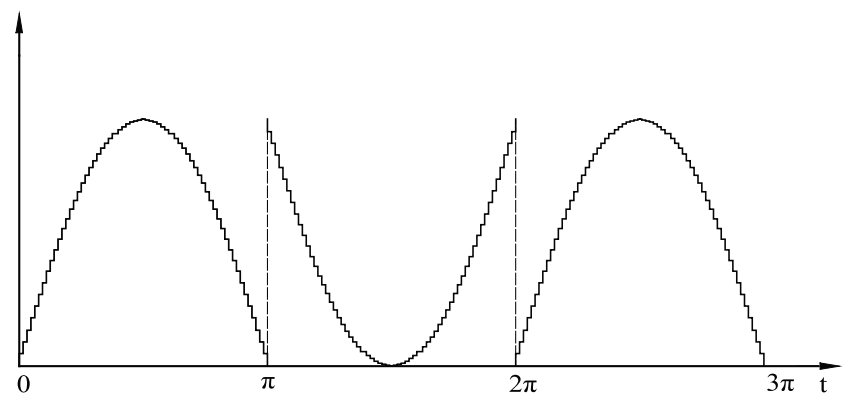

Figure 3. Digital values generation: $\mathrm{f}(\mathrm{x})=\sin \mathrm{x}$ where $0<\mathrm{x}<\pi$, $\mathrm{x}$ takes 66 discrete values, and for $\pi<\mathrm{x}<2 \pi$, $\mathrm{x}$ takes another 66 discrete values

\subsection{Current Synthesis}

The microcontroller's 8-bit output (PortB) is directed to the DAC input. The DAC used in this project was the Texas Instruments THS5641. The THS5641 is an 8-bit resolution digital-to-analog converter and can support up to 100 MSPS (Mega Samples Per Second) update rate. It supports both straight binary and two's complement word format. The THS5641 provides a nominal fullscale differential output current of $20 \mathrm{~mA}$ and greater than $300 \mathrm{k} \Omega$ output impedance, supporting both single-ended and differential applications. The output current can be directly fed to the load (e.g., external resistor load or transformer), with no additional external output buffer required. An accurate on-chip reference and control amplifier allows the user to adjust this output current from $20 \mathrm{~mA}$ down to $2 \mathrm{~mA}$, by just applying the appropriate external resistor $\mathrm{R}_{\mathrm{BIAS}}$, with no significant degradation of performance. In this application the $2.5 \mathrm{~mA}$ was selected.

The THS5641 delivers complementary output currents $\mathrm{IOUT}_{1}$ and $\mathrm{IOUT}_{2}$. Output current IOUT $\mathrm{I}_{1}$ equals the approximate full-scale output current when all input bits are set high in mode 0 (straight binary input), i.e. the binary input word has the decimal representation 255. Full-scale output current will flow through terminal $\mathrm{IOUT}_{2}$ when all input bits are set low (mode 0, straight binary input).

The relation between $\mathrm{IOUT}_{1}$ and $\mathrm{IOUT}_{2}$ can thus be expressed as:

$\mathrm{IOUT}_{1}+\mathrm{IOUT}_{2}=\mathrm{IOUT}_{\mathrm{FS}}$,

where IOUT $_{\mathrm{FS}}$ is the full-scale output current. The output currents can be expressed, in a good approximation, as:

$\mathrm{IOUT}_{1}=\mathrm{IOUT}_{\mathrm{FS}} \times \frac{\mathrm{CODE}}{256}$
$\mathrm{IOUT}_{2}=\mathrm{IOUT}_{\mathrm{FS}} \times \frac{(255-\mathrm{CODE})}{256}$

where CODE is the decimal representation of the DAC data input word provided by the microcontroller.

The digitally controlled analog switch used in this project was the Analog Devices ADG704. The ADG704 is ac-tually a CMOS analog multiplexer, comprising four single channels. The ADG704 switches one of four inputs to a common output, D, as determined by the 3-bit binary address lines, A0, A1 and EN. Each switch of the ADG704 conducts equally well in both directions when ON. The ADG704 exhibits break-before-make switching action. It presents $2.5 \Omega$ (typically) resistance at $5 \mathrm{~V}$ supply. It has very fast switching times $\mathrm{t}_{\text {on }}=20 \mathrm{~ns}$ and $\mathrm{t}_{\text {off }}=15 \mathrm{~ns}$.

The current outputs ( $\left.\mathrm{IOUT}_{1}, \mathrm{IOUT}_{2}\right)$ are connected to $\mathrm{S} 1$ and S3 switch inputs and the output D to the ground. The current outputs are connected to the load resistor as well. The load resistor is usually the sensor element, or device under test (DUT). The programmed selection through the microcontroller switches the appropriate current output to the ground. This is shown in Fig. 4. In this figure, the toggling switch is shown. The two current outputs are summed through this switch, which drains the whole current IOUT $_{\mathrm{FS}}$ to the ground. At any time interval, $\mathrm{I}_{+}$or I flows through the load. From Fig. 4, the alternating nature of the current flow is evident. The two currents $\mathrm{I}_{+}$and $\mathrm{I}_{\text {. }}$ are summed to either node A or node $\mathrm{B}$. This operation ensures that the whole current IOUTFS is drawn from the DAC, as it is required by the manufacturer.
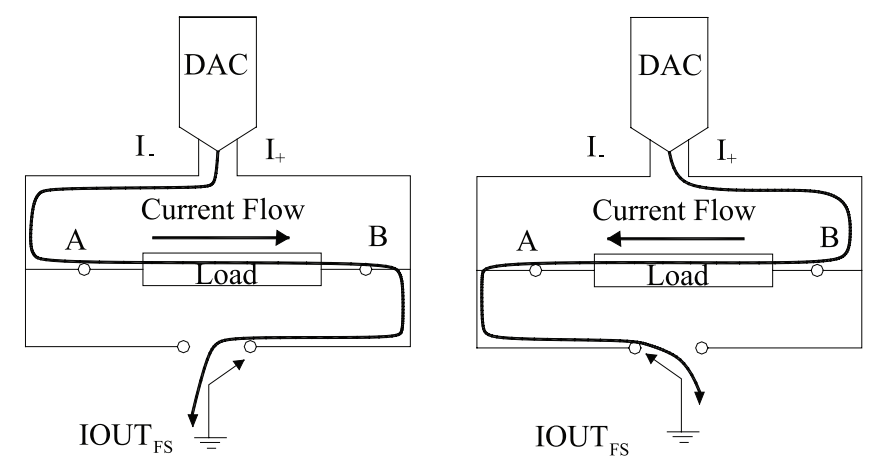

Figure 4. Current flow through the load. The two currents $I_{+}$and I- are summed to either node $\mathrm{A}$ or node $\mathrm{B}$ in order to ensure that the whole current IOUT $_{\mathrm{FS}}$ is drawn from the DAC

\subsection{Microcontroller}

The microcontroller used in the project was the Microchip PIC $16 \mathrm{C} 74 \mathrm{~A}$ and its crystal clock was 4MHz. It is a RISC 8-bit microcontroller and its package at 40pin DIP provides it with flexibility to adapt at any embedded application. The switching action of the analog switch is synchronized with the start and end of each half cycle.

Fig. 5 presents the alternative digital sine program flow chart. The program is written in assembly language and debugged on the RF Solutions ICEPIC (In Circuit Emulator).

\subsection{Evaluation Circuit}

The load resistor leads (A and B in Fig. 4), as before mentioned, 


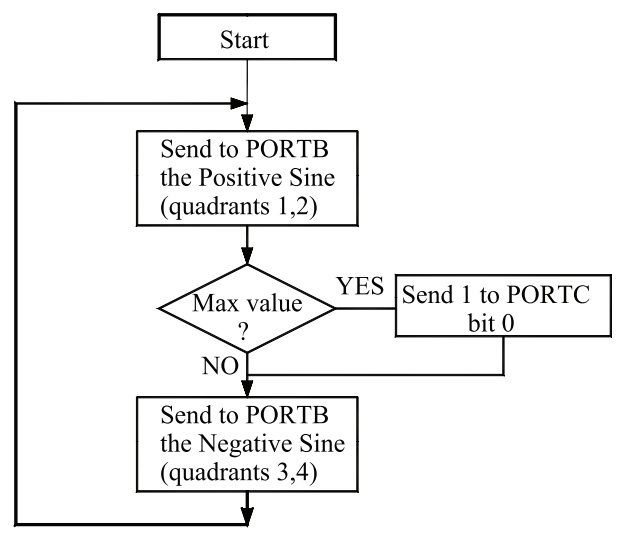

Figure 5. Alternative Digital Sine Flow Chart

are alternatively switched to ground. Thus, it is very difficult to trace the load resistor voltage drop on the oscilloscope. In order to observe the result of this current source, a special circuit was constructed.

For experimental purposes, the two current outputs are directed to a $1 \mathrm{k} \Omega$ load resistor. This load was selected at that value in order to produce the potential difference $1 \mathrm{~V}$ per $\mathrm{mA}$ produced by the DAC. For evaluation purposes, the potential difference is directed to a unity gain instrumentation amplifier (AD622). An oscilloscope traces the instrumentation amplifier's output and photograph 3 shows the alternative sine waveform.

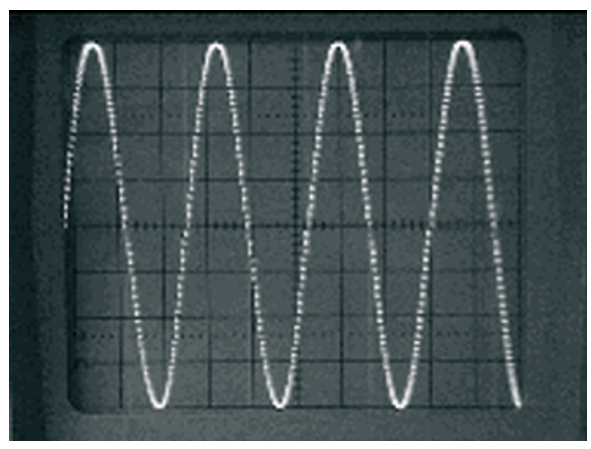

Photo 3. Alternative Sine Waveform

\section{Transfer Function}

The $\mathrm{z}$ transform for a sine function $\mathrm{f}(\mathrm{t})=\mathrm{A} \sin (\mathrm{akT})$ is:

$Y(z)=\frac{A z \sin (a T)}{z^{2}-2 z \cos (a T)+1}$

where $\mathrm{a}$ is a real number, $\mathrm{k}=1,2,3 \ldots$ (natural number) and $\mathrm{T}$ is the sampling step time.

If a pulse input

$\left(X(z)=\frac{z}{z-1}\right)$

is selected as input, then the transfer function of the system producing a digital sinusoidal signal it will be:

$H(s)=\frac{Y(z)}{X(z)}=A \frac{\sin (a T) z^{2}-\sin (a T) z}{z^{3}-2 \cos (a T) z^{2}+z}$
For the current source application, a can be selected to be equal to $\omega=2 \pi \mathrm{f}$ :

$\mathrm{a}=\omega=2 \pi \mathrm{f}=2 \pi 30000=188400 \mathrm{rad} / \mathrm{sec}$ (the sine frequency is $30 \mathrm{kHz}$ )

$\mathrm{T}=3 \mu \mathrm{sec}$ (the sampling step time)

and $\mathrm{A}=2.5 \mathrm{mAmps}$ (the sine amplitude)

So, the transfer function of the produced digital sine is:

$H(z)=2.5 \frac{0.5358 z^{2}-0.5358 z}{z^{3}-1.6886 z^{2} z}$

\subsection{Sampling Error}

A simple reconstruction of a signal generally is defined by:

$f(t)=f\left(t_{k}\right), \quad t_{k} \leq t \leq t_{k+1}$

The signal reconstruction for the selected DAC is zero-order hold $(\mathrm{ZOH})$ and the DAC holds the old value until the next conversion.

If the signal has a continuous second derivative and a periodic sampling then maximum error will be:

$\mathrm{e}_{\mathrm{ZOH}}=\max _{\mathrm{k}}\left|\mathrm{f}\left(\mathrm{t}_{\mathrm{k}+1}\right)-\mathrm{f}\left(\mathrm{t}_{\mathrm{k}}\right)\right| \leq \mathrm{T} \max _{\mathrm{t}} \mathrm{f}^{\prime}(\mathrm{t})$

The $\mathrm{ZOH}$ reconstruction can be considered as an extrapolation with a zero order polynomial.

\section{Synchronous Demodulation}

Assuming that the applied signal is $A \cos (\omega t)$, the measured signal will be [5], [7]:

$V(t)=B \cos (\omega t)+C \cos (\omega t+\varphi)=D \cos (\omega t+\theta)$,

where $\mathrm{B}$ is the amplitude to be measured (real part of the signal), $\mathrm{C}$ is the amplitude of the parasitic signal (imaginary) due to the displacement current through parasitic capacitances between driving and receiving electrodes, and D is the amplitude of the received signal. $\theta$ is the phase difference between the received and the applied signal, and $\varphi$ is the phase difference between the applied and parasitic signal.

A sample of this signal, taken when the applied signal reaches its peak value $(t=0)$, results in an output voltage of the sampling circuit of:

$\mathrm{v}_{0}(\mathrm{dc})=\mathrm{B}+\mathrm{C} \cos \varphi=\mathrm{D} \cos \theta$.

The term $\mathrm{C} \cos \varphi$ contributes an error to the measurement. As the parasitic signal has almost $90^{\circ}$ phase shift, the term $\cos \varphi$ equals to zero. Assuming that in the operating frequency the phase shift becomes $\varphi=90^{\circ}$ (this is the maximum phase shift that a (parasitic) RC circuit can introduce), the input to the sampling circuit will have the magnitude of $\mathrm{B}$ which is the desired:

$\mathrm{v}_{0}(\mathrm{dc})=\mathrm{B}=\mathrm{D} \cos \theta$

This equation gives the peak value of the sinewave. 
The above analysis shows the synchronous behavior of the demodulator unit. For additional explanation, Fig. 6 is given. Apparently, a sample taken when the applied signal crosses zero, provides the imaginary part of the waveform.

In the proposed circuitry the digital sinewave generator gives a pulse corresponding to the zero phase (peak) of the applied signal. This pulse is used to drive the sampling circuit. The sampled values correspond to the real part (in phase) of the received waveform.

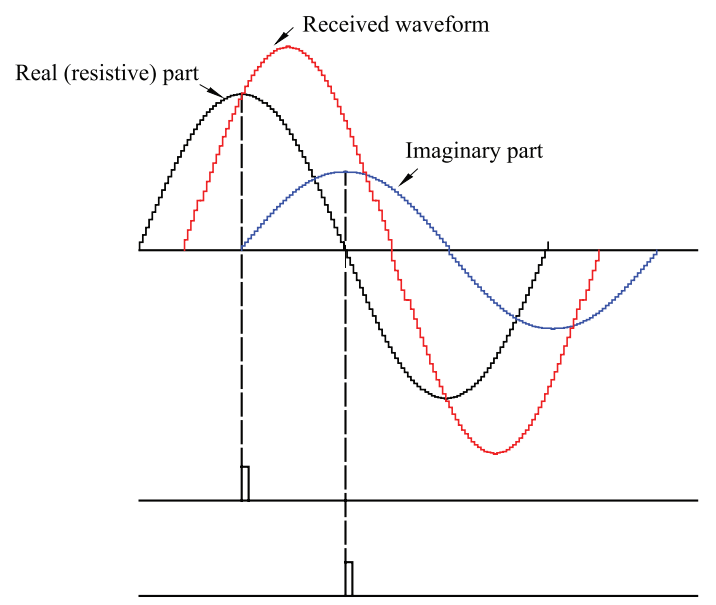

Figure 6. Received waveform decomposed into two waveforms in quadrature. For this case the real (resistive) part is maximum when the imaginary (capasitive) is zero and vice versa

\section{PC Presentation}

A second microcontroller is dedicated to act as data acquisition circuit. It is of the same type as the current source microcontroller, the 16C74A by Microchip. The eight (8) A/D channels it has, have 8-bit resolution.

The microcontroller samples the voltage drop on the load resistance at the time instants directed by the current source microcontroller. That timing is at the positive maximum of the sinusoidal current waveform.

The PC is connected to the data acquisition circuit through the serial RS232 link. A MAX2323 chip is driving the serial bus.

The collected data are serially transferred to the PC. The PC requests the collected data by transmitting the byte «AAh» and the data acquisition microcontroller responds with an 8-bit word. On the PC, a program written in Delphi carries out the appropriate calculations and presents the data on the monitor. Fig. 7 shows the user interface screen on the PC monitor.

The transmitted data are the voltage drop on the load resistance caused by the sinusoidal current, with the $2.5 \mathrm{~mA}$ maximum.

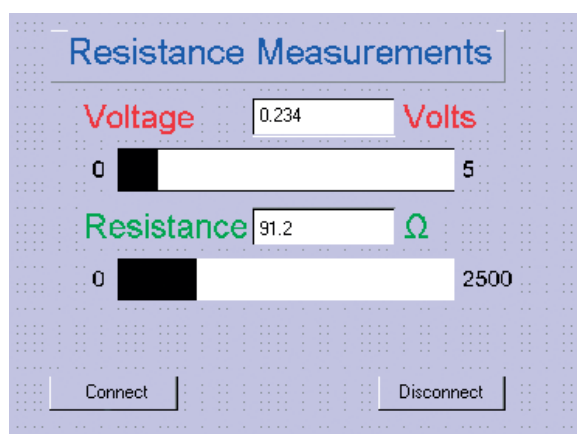

Figure 7. User interface

Since the microcontroller has A/D with 8-bit resolution and a reference voltage of 5 Volts, the data acquisition system has $19 \mathrm{mV}$ resolution. Thus, the resistance resolution that the PC can measure, is $7.6 \Omega$.

\section{Results}

To test the integrated current source - data acquisition system, carbon resistors were selected as load resistance. A $90 \Omega$ carbon resistor was selected and measured on the HP 34401A bench multimeter. The measurement showed an actual resistance of $93.6 \Omega$. This resistor was placed as load and the presented system responded with the value of $91.2 \Omega$. This gives for the measured resistance an absolute error of $2.5 \%$. This error is high, but for higher resistance values the error will minimize. If a $1000.0 \Omega$ resistor is selected (one of many on the self), the measured value would be at $1003.2 \Omega$ and the absolute error at $0.32 \%$.

If the reference voltage on the data acquisition microcontroller is $5 \mathrm{~V}$, the maximum measurable resistance it will be:

$\mathrm{R}_{\max }=\frac{5 \mathrm{~V}}{2.5 \mathrm{~mA}}=2000 \Omega$

We must emphasize here, that the measurement takes place at $30 \mathrm{KHz}$ frequency in contrast to the bench meter which functions at DC.

\section{Conclusions}

This paper has presented a new technique to produce an alternative digitally synthesized sinusoidal waveform using a single power supply. This current source can be applied on sensors to measure the resistance change at frequencies other than $0 \mathrm{~Hz}$ (DC). The data are presented on a PC.

\section{References}

1. Lygouras J. N., Tarchanidis K. N., Tsalides Ph. G., "Suspended Sediment and Dye Concentration Measurements Using a Digital Techniques”, Int. J. Electronics, 87, No.1, pp 107, (2000).

2. Webster J. G. Ed., "Tactile Sensors for Robotics and Medicine", Wiley, New York, (1988).

3. Dally J. W., Rilley W. F., Mcconnel K.G., "Instrumentation for Engineering Measurements", Wiley, New York, (1993).

4. Tarchanidis K. N., Ioannidis I. N., Kakkalis I. K. And Lygouras J. N., "FLEXIMAT, an Integrated System Scanning the Bending of Any
Matress.", Int. Workshop on Virtual and Intelligent Measurement Systems, VIMS 2000, Annapolis, MD, USA, 29-30 April 2000, pp 115, (2000).

5. Taub \& Schilling, "Principles of Communication Systems, $2^{\text {nd }}$ Edition", Mc-Graw Hill, New York, (1987).

6. Koukourlis C. S., Voulgaris N. C., "A Digital Method for Sinewave Generation.” Archiv fur Electrotechnik, 72, pp 327, (1989).

7. Koukourlis Chr. S, Trigonidis V. K., Sahalos J. N., "Differential Synchronous Demodulation for Small Signal Amplitude Estimation.”, IEEE Instrumentation and Measurement, 42, No. 5, pp 926, (1993). 